\title{
Museums, health and wellbeing research: co-developing a new observational method for people with dementia in hospital contexts.
}

\begin{abstract}
:
Aims: The aim of this paper is to present a new observational tool for assessing the impacts of museum object handling for people with moderate-to-severe dementia in hospital settings, focusing on wellbeing, social interaction, level of engagement and agitation. The paper presents a four-step approach to collaboration towards codeveloping the tool, which involved a range of academics, museums professionals, and health and social care partners, and describes the process of integrating multiple perspectives towards common research methodologies.

Methods: The research team organised a series of meetings and workshops with museum and with healthcare partners to identify commonly used assessments and their perspectives on the objectives and possible outcomes of museum object handling activities. These were integrated with findings from a review of current conceptualisations of engagement in people with dementia to produce a fit-for-purpose video evaluation method of the health and wellbeing impacts of the museum object handling programmes.
\end{abstract}

Results: The paper presents the Museum Engagement Observation Tool for use in hospital settings for people with moderate-to-severe dementia.

Conclusion: The paper suggests that collaborative approaches can inform the development of future methods for creative health research and evaluation initiatives and to support this, it outlines the process of development of a new observational tool for people with dementia. 
Key words: wellbeing, dementia, museums and arts for health, collaboration, mixedmethods.

\section{Introduction}

The area of arts in health is currently underdoing growing activity ${ }^{1}$ and has expanded into a range of cultural spaces, most notably, the museum and art gallery sector ${ }^{2}$. Museum-based activities for wellbeing broadly include supported museum visiting, object handling, volunteering, and a range of creative activities inspired from the sector's wide-ranging museum collections, from art making to performing arts and music. In a recent Perspectives paper, Camic and Chatterjee ${ }^{2}$ outline a rationale for the involvement of museums and art galleries as partners in the delivery of health-care interventions and identify practice examples of programmes addressing issues such as dementia $^{3-8}$, mental health ${ }^{9-11}$, cancer $^{12,13}$ and health education ${ }^{14,15}$. Recent studies have also looked at addiction recovery ${ }^{16}$ and older adults at risk of social isolation ${ }^{17,18}$. Museums are increasingly developing services for people with dementia and their caregivers; a recent survey identified that out of over half UK museums offering wellbeing programmes, people with dementia are the second largest target audience (older adults came out top) ${ }^{19}$. Despite this growth in practice there has been limited research to assess the efficacy of museum-led interventions. The paper is based on a research project called 'Not So Grim Up North' (2015-2018), led by University College London researchers in partnership with two northern museum services, the Whitworth Art Gallery and Manchester Museum, The University of Manchester, and Tyne \& Wear 
Archives \& Museums. This was a multi-sited project with multiple health and social care partners, exploring the health and wellbeing impact of taking part in cultural activities, focused on three audience groups: mental health service-users, stroke survivors and people with dementia. The research was designed as a quasi-experimental study using mixed-methods to address multiple perspectives and multi-level approaches to the question of impact (Health Research Authority Ethics ID 199643). One strand of the research focused on object handling sessions for people with moderate-to-severe dementia using the museums' handling collection, predominantly everyday social history objects from the 1950-1970s. The object handling focused on a non goal-oriented activity as opposed to reminiscence, promoting sensory engagement and conversation at a level appropriate for people with moderate-to-severe dementia, in line with recommendations from other non-reminiscence interventions focused on the benefits of cognitive stimulation for dementia ${ }^{20}$. This paper focuses on the codevelopment of the methodological approach to assessing engagement in these sessions and its impact.

The paper will first briefly discuss the concept of engagement in people with dementia and its application in our study. Next, the paper will outline a four-step approach to collaboration conducted in this study. It will then describe how multiple stakeholder perspectives, including museum and healthcare professionals, were integrated towards developing a common impact-focused methodology. The final section will present a new observational method for people with dementia in hospital settings developed in the course of the study. The paper will conclude with a consideration of the wider application of a collaborative approach for projects researching creative and cultural interventions for health. 


\section{Engagement in people with dementia}

Engaging a person with dementia in meaningful activity is seen as a priority in care provision to promote wellbeing and quality of life ${ }^{21-23}$. Studies in care homes have shown the adverse effect of prolonged lack of stimulation and inactivity, such as increased risk of cognitive decline, apathy, depression and agitation ${ }^{24,25}$, and the positive impact of participation in activities, which can lead to a reduction in challenging behaviours and depression, and improvements in mood $1222,26,27$. The study of engagement is therefore fundamental to the development of non-pharmacological interventions for people with dementia ${ }^{28}$ and to determine the efficacy of interventions that promote meaningful activity ${ }^{29}$. Examples of meaningful activity include ${ }^{21}$ : groups organised for peer support, life story work, intergenerational activities, Montessoribased activities, new creative activities (such as music, dance and arts activities), reminiscence and memory-recall activities, Cognitive Stimulation Therapy ${ }^{30-32}$ (CST), as well as re-acquaintance with previously conducted activities. It has been proposed that museum activities, including museum visits and object handling sessions, are another example of a potential meaningful activity for people with dementia and their

carers. Recent studies reported positive wellbeing impact for people with mild-tomoderate dementia taking part in creative museum activities with their carers, including museum object handling and art viewing in a gallery ${ }^{7,8}$. A further study defined the environmental attributes of the art gallery and their positive emotional and relational effects for people with dementia and their carers ${ }^{6}$.

The needs and abilities of people change with the various stages of dementia and therefore activities should be adapted across the dementia trajectory. A recent study examined the characteristics of activities in relation to the stages of dementia ${ }^{33}$. The 
authors found that people with mild dementia could readily engage with complex arts and crafts activities and cognitive activities requiring multi-steps. People with moderate dementia engaged with activities that were not necessarily goal-orientated, requiring repetitive tasks, with one or two-steps directions, and people with severe dementia engaged with simple non goal-oriented activities such as simple movement tasks and/or manipulation/sensory/sorting activities. Museum object handling can therefore be adapted across the dementia trajectory, from being part of complex creative activities to more simple tactile engagement. Our study aims to examine the experience of engagement with museum objects in the context of a handling session delivered in a hospital ward for people with moderate-to-severe dementia. The sessions are framed as a meaningful activity adapted to the level of dementia, and as a potential adjunct to approaches such as CST.

\section{Collaborative research for co-developing methodologies}

Previous research has highlighted the importance of conducting collaborative research into the efficacy of engagement activities for people with dementia and their carers. The REMCARE study undertook a Randomised Controlled Trial (RCT) to assess the effectiveness and cost-effectiveness of group reminiscence for people with dementia and caregivers $(n=350)^{34}$. Reminiscence is one of the most widely deployed engagement activities for people with dementia, but research regarding the efficacy of reminiscence and other memory recall activities has revealed mixed findings. The REMCARE study found no significant differences in outcomes (including Quality of Life and GHQ-28) between the intervention and control conditions and a significant increase in carer burden, anxiety and stress. Notwithstanding these findings, RCTs are 
expensive and may not capture the nuanced, more qualitatively-derived advantages of cultural engagement activities designed for people with dementia. Our argument in this paper is that all research projects should begin with consultation of key groups and that there are particular advantages to co-developing methods to investigate the effects and impact of a cultural intervention. In the following section, we briefly summarise our approach to co-developing methodologies as a 4 step-approach that can be applied to other arts in health and museums in health programmes (see figure 1).

Step 1: Identifying benefits from multiple perspectives. In order to understand the benefits of cultural programming for health, it is crucial to understand the situated contexts of each project partner and participant. This ensures that research is grounded in the experiences of those people who are at the centre of the study. Research that builds on experience is especially important when the objective is to evaluate impact. We suggest it is also useful to understand commonly used forms of assessment in healthcare settings to further elucidate how impact is framed and measured in terms of health and wellbeing outcomes and from the perspectives of healthcare staff. Interviews (formal and informal) and focus group discussions can be used to identify areas of benefits. Questions should be directed to uncover stakeholders' perspectives on the objectives and possible outcomes of creative activities. Additionally, informal feedback can be used with clients and patients during the pilot sessions to capture a sense of their experience and their own understandings of what supports their health and wellbeing.

Step 2: Integrating multiple perspectives and co-developing methodologies. The aim is to integrate diverse ways of knowing the impact of cultural programmes, bringing together experiential knowledge, patient values, professional judgements and outcomes-based assessments, in order to co-develop a methodology to capture this 
evidence. It should be noted that the development of new methods is an iterative and collaborative process, and therefore requires multiple research meetings to refine and agree the final methodology.

Step 3: Implementation, refers to the study set up, recruitment and data collection. This step should also include pilot sessions, allowing for methods to be tested and further refined. In many cases the academic research team will conduct the data collection, but this step might also include research partners to collect other information, whether clinical, observational or reflective.

Step 4: Interpretation, is the data analysis and interpretation of findings. Often this phase is undertaken by the academic research team. Within the principles of collaborative research, it is also important to factor in time to share with partners both the findings in a digestible form as evidence of the impact of the project, as well as the learning applicable to project delivery. This can ensure that learning is incorporated into future programme design and delivery .

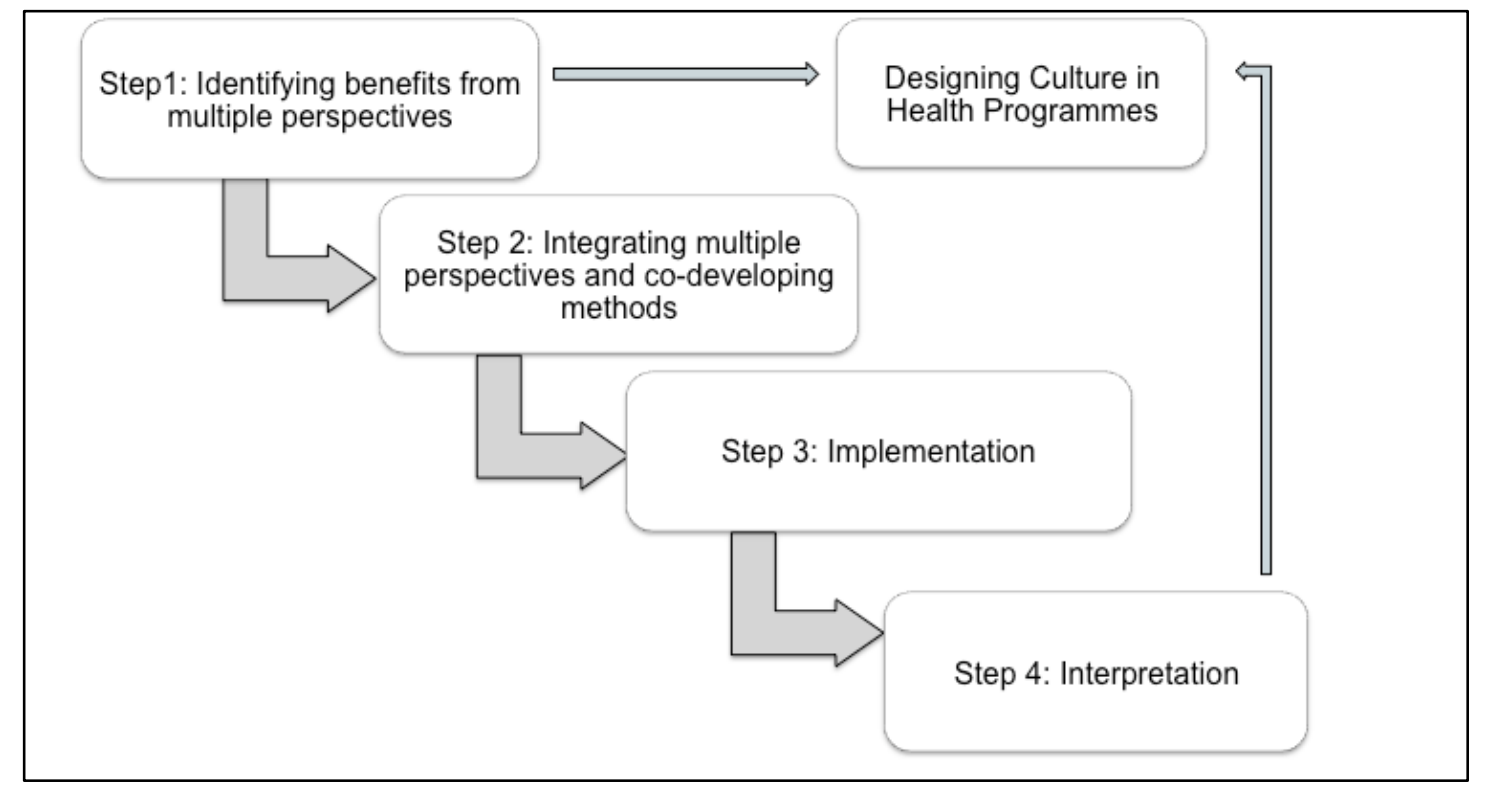

Figure 1: Co-developing methodologies for culture in health projects: a 4-step approach 


\section{Understanding multiple perspectives and commonly used assessments in a dementia care hospital unit}

The context for the research strand on dementia was a museum project developed for people with moderate-to-severe dementia in a specialised inpatient dementia unit. The service works as a 24-hour setting with a focus on psychosocial approaches to assess and manage behaviours that challenges in people with dementia ${ }^{35}$. Typically, patients are assessed over a six-week period during which a care plan is developed. The museum programme was developed as a weekly 1 -hour session, over a six-week period. The first phase of the study was to understand the perspectives of different individuals involved in the delivery and support of museum project and those directly engaged in the activities.

The academic team first organised a number of research meetings with museum partners to discuss their programme aims and explore how they framed the anticipated outcomes for participants. The museum professionals wanted the programme to support participant wellbeing by providing an enjoyable time and an opportunity to connect emotionally with museum objects they might remember from their childhood. The aim here was not framed as reminiscence but rather as a meaningful activity for pleasure, cognitive stimulation and to spark conversation with others. A related second aim of the project was to encourage social interaction between patients, and between patients and care staff. 
Next, several research meetings were facilitated by the research team with care staff at the hospital to further develop the project and to identify how patients might benefit from engaging in the sessions, thinking about the hospital teams' specific care priorities for this patient group. The meetings included ward staff, an activity coordinator and an occupational therapist. For these staff, the approach to care is person-focused, a key aspect of which is to formulate activities that are 1) personally meaningful to the individual (i.e. linking to a personal interest or aspects of their personality) and 2) effective in terms of the individual's level of ability to engage in the activity. Such activities were aimed at creating enabling environments for individuals and to manage behaviours that challenge. The museum activities were framed to fit within this model to address patient mood and agitation (if present), provide positive cultural engagement that would capture patients' attention, and support positive social interaction with other patients on the ward. Staff further reiterated the need for a non-obstructive method of data collection as interviews and questionnaires would not be appropriate for patients who are more seriously ill or cognitively impaired. Due to the particular nature of the ward, it was not possible to include the perspectives of carers and family members.

The research team also worked with healthcare staff to identify currently used assessment to measure these aspects of care, with the aim of investigating the benefits of the museum sessions in the terms of care staff's working practices, knowledge and perspectives, and considering the suitability and relevance of routinely used measures for application to the museum activities. The conversations with healthcare staff indicated that no tools were consistently used to assess the impact of ward-based nonclinical activities. Instead, bespoke assessment tools were used or tools were adapted for record keeping, such as the Pool Activity Level (PAL) Instrument for Occupational 
Profiling ${ }^{36}$. While the adapted PAL tool was not appropriate for the study as it focuses on assessing a patients' level of ability, the 10-item wellbeing element was identified by staff as being more useful and detailed for describing patient's mood than shorter scales. A series of pilot sessions were also conducted at the hospital between JulyAugust 2016 and were observed by a researcher.

\section{Results: The Museum Engagement Observation Tool for PWD}

The observations of the pilot sessions and research conservations with staff and museum facilitators were integrated to identify the key areas of direct benefit of participants with moderate-to-severe dementia in museum object handling activities,. These were collaboratively agreed as: attention (attitude towards the session), engagement (attitude towards the museum object), social interaction, wellbeing and agitation (presence or absence). This research led to the development of an experimental scale. An observational approach was chosen for this study as it can be used regardless of the level of cognitive impairment of participants observed. Video analysis was chosen as way to enable continuous observation.

From a review of published literature, two studies were identified as useful frameworks for conceptualising the different dimensions of engagement with museum objects: the Observation Method of Engagement ${ }^{28}$ (OME) and the Video Coding- Incorporating Observed Emotion $^{29}$ (VC-IOE). Both are based on the conceptual framework for engagement developed by Cohen-Mansfield et al. ${ }^{28}$ The OME was developed specifically to examine engagement for people with dementia in residential care and measures five dimensions of engagement: 1) Rate of refusal of the stimulus; 2) Duration 
of time that the resident is occupied with the stimulus; 3) Level of attention to the stimulus (4-point scale from 'not attentive' to 'very attentive'); 4) Attitude to the stimulus (7-point scale from 'very negative' to 'very positive'); 5) Action towards the stimulus, such as holding it or talking to it (4-point scales ranging from 'none of the time' to 'most of the time'). The VC-IOE developed from this work as a tool for video coding in the context of engagement with social robots in long-term residential care homes. It focuses on the duration of six dimensions of engagement: emotional (based on a modified version of the Observed Emotion Rating Scale ${ }^{37}$ ), verbal, visual, behavioural, collective engagement, which are assessed as either positive, negative or absent; and signs of agitation (evident or not evident).

The Museum Engagement Observation Tool was created to meet the needs identified by the research partners and is based on the concepts underpinning these two observation methods. It was developed to capture the distinctive nature of engagement with museum objects, and was revised and refined over six months in collaboration with healthcare staff, in particular the operational definitions and rating scales (Figures $2 \& 3)$.

The Museum Engagement Observation Tool measures five dimensions of engagement within a museum object handling session:

- Attention refers to the level of concentration of participants towards the museum activity, from 'unable to concentrate' to 'concentrates for full periods'. - Engagement refers to the attitude towards the object, based on a re-worked version of the OME and VC-IOE. Engagement is understood as 'the act of being occupied or involved with an external stimulus' following the definition by 
Cohen-Mansfield et al. ${ }^{28}$. Whereas the VC-IOE separates visual, verbal and behavioural engagement into three distinct categories, in the Museum Engagement Observation Tool they are combined to produce a shorter scale. It contain five items that describe the level of engagement with the object, from more passive visual engagement or passive handling responding to prompts to higher levels of engagement where the participants explore museum objects in a self-directed manner and where the object sparks discussion. The items were developed to consider the specific value of a museum object handling session, but could be revised for other arts in health projects thinking about the specific properties of the arts activity.

- Social Interaction between participants and the facilitator, and with other participants. It includes 4 items describing different levels of interaction and towards whom the interaction is directed.

- Wellbeing is based on the five different dimensions of affect from the Observed Emotion Rating Scale ${ }^{37}$ (OERS) which is integrated in the VC-IOE. However, some of the wording was changed on the advice of healthcare staff (see discussion above): pleasure is described as 'shows enjoyment' and general alertness is described as 'bright, reactive'; other words were changed to include a wider spectrum of emotions together: anger is described as 'angry/irritable' and sad is described as 'sad/low mood'. Other categories are included to reflect the discussion with staff, including shows humour, resting/settled, asleep, and an 'other (please state)' category. To simplify this for analysis, this element of the scale can be grouped into three dimensions of positive, negative and neutral affect. 
-Agitation examines the presence or absence of signs of agitation, based on the consensus definition by Cummings et $\mathrm{al}^{38}$.

A sixth domain, address, records whether the museum facilitator or a member of staff is directly engaging the person in the session, to gauge the level of support required within the museum sessions.

[Insert Figures 2 and 3 here]

The tool was developed for direct observation within sessions and for use in video analysis. During the study, observations were taken by the researcher at the beginning, middle and end of a one-hour session, with each participant observed for total of one minute. The tool is subsequently being used for continuous video analysis, including establishing test-retest reliability of the tool; results of these studies will be reported separately.

\section{Concluding comments}

This article presents a new observational tool to investigate the distinctive value of museum object handling programmes for people with dementia. Given that museums and galleries are increasingly working with people with dementia and carers as a key target audience, it is essential to characterise and assess the efficacy of these activities. In future articles we intend to describe the relationship between engagement, object attributes, social interaction and agitation in people with dementia. Situated as a potential adjunct to approaches to such as Cognitive Stimulation Therapy, museum 
object handling as a meaningful activity for people with dementia has considerable potential to take advantage of cultural assets such as museums and art galleries, and develop a new suite of cultural engagement activities for people with dementia. This article lays the methodological groundwork for such investigations.

This paper also sought to summarise our approach to collaboration and present a four step approach for co-developing methodologies for research in museums/arts for health. This is based on the belief that higher quality, more useful and relevant research results from collaboration and from integrating the diverse perspectives of multiple stakeholders.

\section{References}

1. Stickley T, Parr H, Atkinson S, et al. Arts, health \& wellbeing: reflections on a national seminar series and building a UK research network. Arts \& Health 2017; 9: 14-25.

2. Camic PM, Chatterjee HJ. Museums and art galleries as partners for public health interventions. Perspectives in Public Health 2013; 133: 66-71.

3. MacPherson S, Bird M, Anderson K, et al. An art gallery access programme for people with dementia: 'you do it for the moment'. Aging Ment Health 2009; 13: $744-752$.

4. Eekelaar C, Camic PM, Springham N. Art galleries, episodic memory and verbal fluency in dementia: An exploratory study. Psychology of Aesthetics, Creativity, and the Arts 2012; 6: 262-272.

5. Rosenberg F. The MoMA Alzheimer's Project: Programming and resources for making art accessible to people with Alzheimer's disease and their caregivers. Arts \& Health 2009; 1: 93-97. 
6. Camic PM, Baker EL, Tischler V. Theorizing How Art Gallery Interventions Impact People With Dementia and Their Caregivers. The Gerontologist 2015; Online First.

7. Camic PM, Hulbert S, Kimmel J. Museum object handling: A health-promoting community-based activity for dementia care. Journal of Health Psychology 2017; 1359105316685899.

8. Johnson J, Culverwell A, Hulbert S, et al. Museum activities in dementia care: Using visual analog scales to measure subjective wellbeing. Dementia 2015; 1471301215611763.

9. Shaer D, Beaven K, Springham N, et al. The role of art therapy in a pilot for artbased Information Prescriptions at Tate Britain. International Journal of Art Therapy 2008; 13: 25-33.

10. Roberts S, Camic PM, Springham N. New roles for art galleries: Art-viewing as a community intervention for family carers of people with mental health problems. Arts \& Health 2011; 3: 146-159.

11. Ander E, Thomson LJ, Blair K, et al. Using museum objects to improve wellbeing in mental health service users and neurological rehabilitation clients. The British Journal of Occupational Therapy 2013; 76: 208-216.

12. Lanceley A, Noble G, Johnson M, et al. Investigating the therapeutic potential of a heritage-object focused intervention: a qualitative study. J Health Psychol 2012; 17: 809-820.

13. Thomson LJ, Ander EE, Menon U, et al. Enhancing Cancer Patient Well-Being With a Nonpharmacological, Heritage-Focused Intervention. Journal of Pain and Symptom Management 2012; 44: 731-740.

14. Wikström BM. Nursing education at an art gallery. Journal of Nursing Scholarship: An Official Publication of Sigma Theta Tau International Honor Society of Nursing 2000; 32: 197-199.

15. Wikström B-M. Works of art as a pedagogical tool: an alternative approach to education. Creative Nursing 2011; 17: 187-194.

16. Morse N, Thomson LJM, Brown Z, et al. Effects of creative museum outreach sessions on measures of confidence, sociability and well-being for mental health and addiction recovery service-users. Arts \& Health 2015; 7: 231-246.

17. Solway R, Camic PM, Thomson LJ, et al. Material objects and psychological theory: A conceptual literature review. Arts \& Health 2015; 0: 1-20.

18. Thomson LJM, Chatterjee HJ. Well-Being With Objects: Evaluating a Museum Object-Handling Intervention for Older Adults in Health Care Settings. Journal of Applied Gerontology 2016; 35: 349-362. 
19. Lackoi K, Patsou M, Chatterjee HJ. Museums for Health and Wellbeing. A preliminary report.https://museumsandwellbeingalliance.wordpress.com/ (2016, accessed 31 October 2016).

20. Aguirre E, Woods RT, Spector A, et al. Cognitive stimulation for dementia: a systematic review of the evidence of effectiveness from randomised controlled trials. Ageing Res Rev 2013; 12: 253-262.

21. Nyman SR, Szymczynska P. Meaningful activities for improving the wellbeing of people with dementia: beyond mere pleasure to meeting fundamental psychological needs. Perspect Public Health 2016; 136: 99-107.

22. van der Ploeg ES, Eppingstall B, Camp CJ, et al. A randomized crossover trial to study the effect of personalized, one-to-one interaction using Montessori-based activities on agitation, affect, and engagement in nursing home residents with Dementia. International Psychogeriatrics 2013; 25: 565-575.

23. Materne CJ, Luszcz MA, Goodwin-Smith I. Increasing constructive engagement and positive affect for residents with severe and very severe dementia through group-based activities. Australasian Journal on Ageing 2014; 33: E7-E10.

24. Cacioppo JT, Hughes ME, Waite LJ, et al. Loneliness as a specific risk factor for depressive symptoms: cross-sectional and longitudinal analyses. Psychol Aging 2006; 21: 140-151.

25. Zuidema S, Koopmans R, Verhey F. Prevalence and Predictors of Neuropsychiatric Symptoms in Cognitively Impaired Nursing Home Patients. Journal of Geriatric Psychiatry and Neurology. Epub ahead of print 29 June 2016. DOI: 10.1177/0891988706292762.

26. Brooker DJ, Woolley RJ, Lee D. Enriching opportunities for people living with dementia in nursing homes: an evaluation of a multi-level activity-based model of care. Aging Ment Health 2007; 11: 361-370.

27. Gitlin LN, Winter L, Burke J, et al. Tailored Activities to Manage Neuropsychiatric Behaviors in Persons With Dementia and Reduce Caregiver Burden: A Randomized Pilot Study. The American Journal of Geriatric Psychiatry 2008; 16: 229-239.

28. Cohen-Mansfield J, Dakheel-Ali M, Marx MS. Engagement in persons with dementia: the concept and its measurement. Am J Geriatr Psychiatry 2009; 17: 299-307.

29. Jones C, Sung B, Moyle W. Assessing engagement in people with dementia: a new approach to assessment using video analysis. Arch Psychiatr Nurs 2015; 29 : 377-382.

30. Spector A, Thorgrimsen L, Woods B, et al. Efficacy of an evidence-based cognitive stimulation therapy programme for people with dementia: randomised controlled trial. Br J Psychiatry 2003; 183: 248-254. 
31. Spector A, Orrell M, Woods B. Cognitive Stimulation Therapy (CST): effects on different areas of cognitive function for people with dementia. Int J Geriatr Psychiatry 2010; 25: 1253-1258.

32. Spector A, Gardner C, Orrell M. The impact of Cognitive Stimulation Therapy groups on people with dementia: views from participants, their carers and group facilitators. Aging Ment Health 2011; 15: 945-949.

33. Regier NG, Hodgson NA, Gitlin LN. Characteristics of Activities for Persons With Dementia at the Mild, Moderate, and Severe Stages. Gerontologist; Online First. Epub ahead of print 2016. DOI: 10.1093/geront/gnw133.

34. Woods RT, Bruce E, Edwards RT, et al. REMCARE: reminiscence groups for people with dementia and their family caregivers - effectiveness and costeffectiveness pragmatic multicentre randomised trial. Health Technol Assess 2012; 16: v-xv, 1-116.

35. James IA. Understanding behaviour in dementia that challenges: a guide to assessment and treatment. London: Jessica Kingsley, Jessica Kingsley Publishers, 2011.

36. Pool J. The Pool Activity Level (PAL) Instrument for Occupational Profiling: A Practical Resource for Carers of People with Cognitive Impairment Fourth Edition. London: Jessica Kingsley Publishers, 2012.

37. Lawton MP, Van Haistmas K, Klapper JA. Observed Emotion Rating Scalehttps://www.abramsoncenter.org/media/1199/observed-emotion-ratingscale.pdf (1999, accessed 12 April 2017).

38. Cummings J, Mintzer J, Brodaty H, et al. Agitation in cognitive disorders: International Psychogeriatric Association provisional consensus clinical and research definition. Int Psychogeriatr 2015; 27: 7-17. 


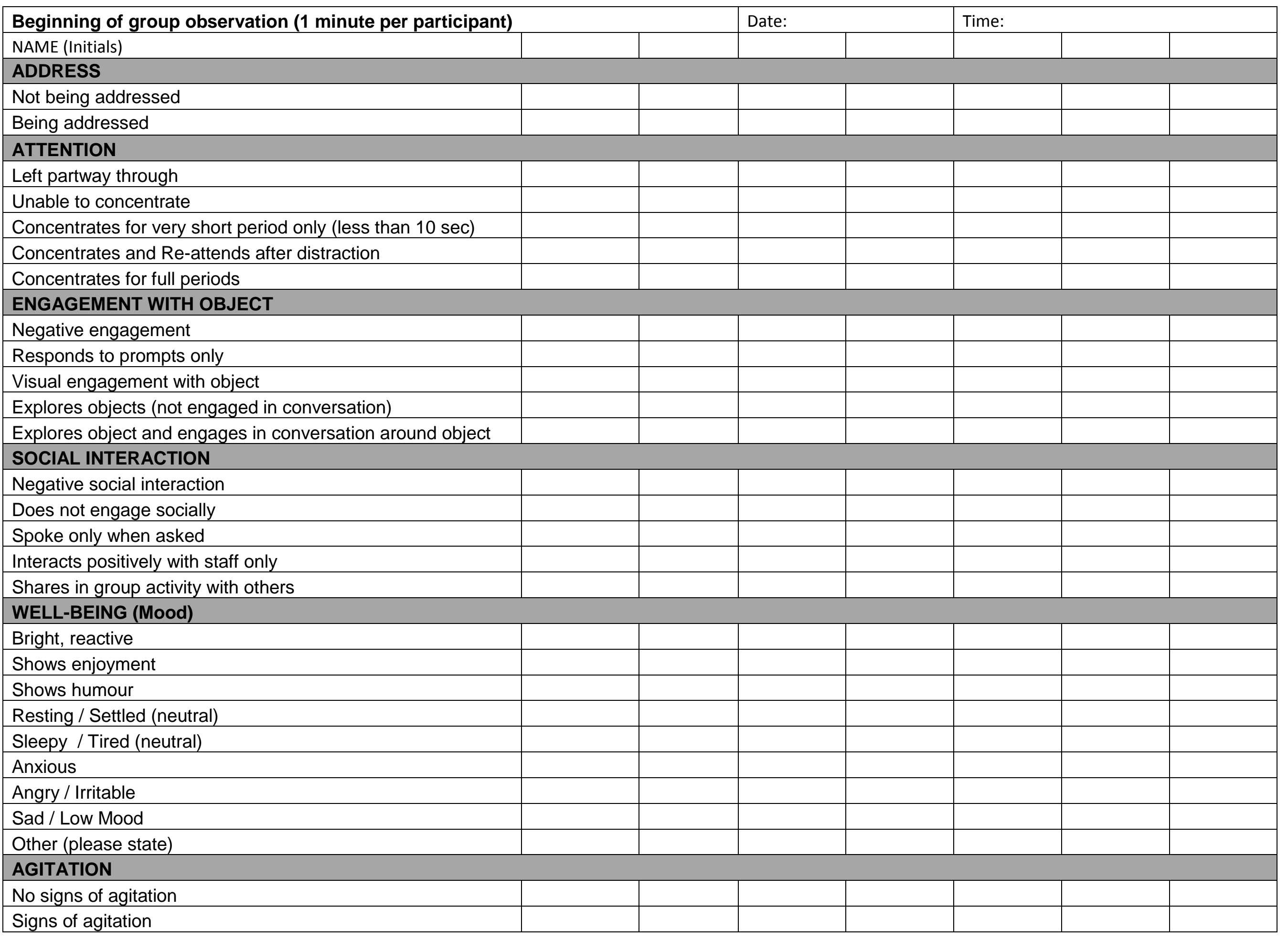




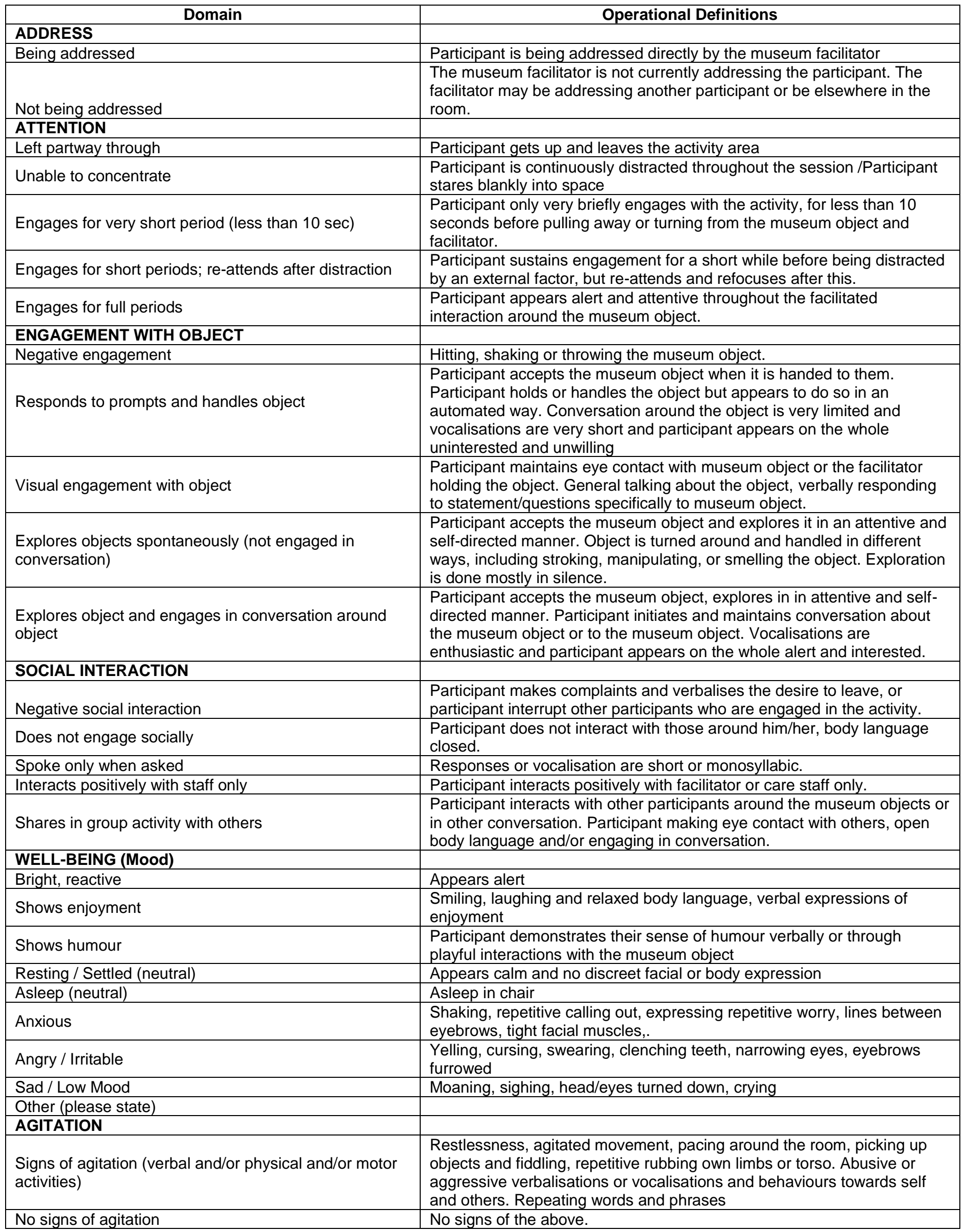

Figure 3: Operational Definitions of the Museum Engagement Observation Tool for PWD 